notions? His favoured option, carefully argued against other candidates, is "The Syntactic Theory of Mind"' (STM). It is introduced in these words:

The basic idea of the STM is that the cognitive states whose interaction is (in part) responsible for behaviour can be systematically mapped to abstract syntactic objects in such a way that causal interactions among cognitive states, as well as causal links with stimuli and behavioura events, can be described in terms of the syntactic properties and relations of the abstract objects to which the cognitive states are mapped. More briefly, the idea is that causal relations among cognitive states mirror formal relations among syntactic objects. If this is right, then it will be natural to view cognitive state tokens as tokens of abstract syntactic objects.

I do not find this altogether intelligible, and not just because I fail to understand how objects can have a syntax. I do not really grasp how a theory such as this (however elaborated) could illuminate mental processes, and particularly the ways in which they go wrong in real life. However, it will be retorted (rightly) that the theory is not really a theory at all, but a kind of prolegomenon for a cognitive theorizing. One might suppose, then, that it is meant to be a methodological prerequisite for research, on the analogy with the methodologies for behaviourism which were proposed in the 1940 s and 1950s. But there seems to me an interesting difference between them. The philosophers of science who analysed the concepts of behaviourism, as well as the concepts of the physical sciences, based their research on the use of the concepts within the theories. Philosophers of mind tend to ignore previous theory and appeal to their own insights, conjectures and intuitions, paraded forth in a series of pregnant sentences. There is nothing wrong with this, and it is often quite effective in advancing an argument. But the logical precision achieved in this way is also likely to be much more puzzling to the non-specialist than was the more discursive work of some of the great philosophers of the past.

There is a real danger that philosophers will end up talking only to each other and abandon their traditional role of protecting us from conceptual confusion. My doubt about the present book is whether its thesis will be of interest to experimentalists. It is a doubt rather than a verdict. Although Stich's arguments are nearly always compellingly precise, I am unsure whether they are consequential, or merely dazzling moves in an intellectual game. In the nature of things, they will not be readily settled because assertions about states of mind are not to be verified like assertions about nerve impulses. So while this book may be of interest to philosophers and to cognitive scientists, it remains uncertain whether it will help the rest of us in trying to find out more about mental processes.

Peter Wason is Reader in Psycholinguistics at University College London. With P.N. Johnson-Laird he is co-author of Psychology of Reasoning: Structure and Content (Batsford, 1972).

\section{Talk of research}

\section{Augustine Brannigan}

Opening Pandora's Box: A Sociological Analysis of Scientists' Discourse.

By G. Nigel Gilbert and Michael Mulkay. Cambridge University Press: 1984. Pp.202. Hbk £22.50, \$37.50; pbk £7.95, \$12.95.

OVER the past decade, a close descriptive approach to scientific behaviour has revived interest in the cognitive and social dimensions of contemporary scientific knowledge. Following in this direction, the Pandora investigation focused on a recent controversy in biochemistry. The study is based on intensive interviews with 34 bioenergists in the United States and Britain, as well as on a sample of their correspondence and a reading of the literature in the field between 1960 and 1980 . This period witnessed the rise of competing theories to explain how the crucial ATP molecule was able to move and temporarily store energy in living cells. In 1978 Peter Mitchell was awarded a Nobel Prize for chemistry for his work on the role of oxidative phosphorylation in ATP formation.

However, research by the authors indicated that members of the "ox-phos" community, as it is called, were far from in agreement about what exactly had been discovered in chemiosmotic research, about the empirical validity of the various bits of evidence, and about the respective parts played by the leading contributors. In their travel between the various biochemical laboratories, Gilbert and Mulkay report they were struck by the fact that although they realized that they were being given radically different accounts by participants of what "really" had happened, each account seemed, on its own, plausible and convincing.

Rather than trying to find out who was "really" correct and who was in error, the sociologists, not being in the business of second-guessing biochemical questions, turned their attention to what they describe as "discourse analysis". This is what opens Pandora's Box. Discourse analysis requires that the sociological observer remains neutral as to who is "actually" correct, the object being to determine how the respective participants produce and maintain their own sometimes contradictory versions of reality in the face of what would seem to be a set of invariant natural facts.

The Pandora investigation was not undertaken as a critique of science, for Gilbert and Mulkay go well beyond observations of how scientists selectively expose themselves to natural facts and selectively perceive and retain them. The heart of their inquiry is the observation that scientists employ two repertoires or codes of discourse for talking about how nature is discovered. The familiar empiricist frame of mind explains progress as following nat- urally from adherence to careful observation, and the testing of rival hypotheses in controlled experiments. In addition to this, scientists also employ what is called a "contingent" repertoire in which progress is traced to unique, subjective or situationally specific factors. Gilbert and Mulkay illustrate the use of these two conflicting repertoires in a fascinating contrast between the informal factors that influenced the investigation and interpretation of "ox-phos" experimental data reported to them in interviews, and the formal rationality provided in the earlier published articles of the same researchers. Rather than siding with the informal "inside" story as the true one, they instead stress that the accounts given of actions vary with the needs of the circumstance in which they are produced. Different accounts draw from the original experience in different ways depending on the formality or informality of the situation.

Having made a convincing case for the existence of two separate repertoires for reporting the progress of chemiosmotic theory, the authors then go on to suggest that these repertoires are employed to explain the differential acceptance of scientific beliefs across the community. While one would expect that consensus ought to follow closely the reading of the facts, this is not the case. The evidence shows that scientists typically ground their own beliefs in empiricist discourse, as following from the experimental results, while explaining the position of opponents in the contingent repertoire: opponents wrongly interpret the facts because of subjective bias, experimental incompetence, theoretical ignorance - and even national origins! This switching between the two repertoires virtually guarantees that opponents will talk past one another. According to the authors, the device which saves the community from solipsism is the belief that in the end "the truth will out" though how, when and in whose favour, no one can determine beforehand.

Other highlights of this work include an outstanding analysis of the tension between the virtual versus the fictional elements in scientific drawings of the chemiosmotic mechanisms. One informant dubs them "working conceptual hallucinations". There are also discussions of consensus and the significance of scientific humour. Each of these topics is explored usefully in the context of the tworepertoire dichotomy. While based on a single scientific speciality, the lessons of this investigation probably have wide currency in all areas of science, especially where controversy mobilizes the two incommensurable structures of discourse identified here. For that reason the book will be read with profit by practising scientists as well as by sociologists of scientific knowledge.

Augustine Brannigan is Associate Professor of Sociology at the University of Calgary, Alberta. 